\title{
Immunonkologie im Fokus
}

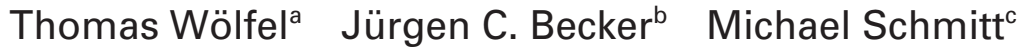 \\ a III. Medizinische Klinik und Poliklinik (Hämatologie, internistische Onkologie, Pneumologie), Universitätsmedizin Mainz, Deutschland \\ ${ }^{\mathrm{b}}$ Klinische Abteilung für allgemeine Dermatologie, Medizinische Universität Graz, Österreich \\ c Siebeneicher-Stiftungsprofessor für Zelluläre Immuntherapie, Medizinische Klinik V, Universitätsklinikum Heidelberg, Deutschland
}

\section{Immunonkologie: Grundlagen für den therapeutischen Einsatz}

Die Antigenerkennung durch zytotoxische T-Lymphozyten (cytotoxic T lymphocytes, CTL) ist ein komplexer Prozess und wurde bereits in den 1980er-Jahren entdeckt [1]. Mittlerweile sind die einzelnen Schritte von der Expression über die Prozessierung und Komplexierung bis zur Interaktion zwischen der antigenpräsentierenden Zelle (antigen-presenting cell, APC) und der T-Zelle mit ihren Rezeptoren (T cell receptors, TCRs) genauer aufgeklärt.

\section{Immunaktivierung}

Antigene werden durch dendritische Zellen, Makrophagen und B-Zellen erkannt und mittels Endozytose aufgenommen. Präsentiert werden die Antigene von diesen spezialisierten Immunzellen, indem aus ihnen prozessierte Peptide an Moleküle des humanen Leukozytenantigen-Systems (human leukocyte antigen system, HLA-System) binden. Fremde oder abnorme, z.B. mutierte Peptide werden von spezifischen TCRs auf der Oberfläche von APCs erkannt. Obwohl durch Umlagerung und Expression der Gene für die $\alpha$ - und $\beta$-Ketten des TCR zirka $10^{18}$ verschiedene $\alpha \beta$-TCRs entstehen, reicht diese Menge nicht aus, um für jedes Antigen einen passenden Rezeptor bereitzustellen. Aus diesem Grunde genügen für die Erkennung eines Antigens Ähnlichkeiten. Diese Ähnlichkeiten haben jedoch das Potenzial, beim therapeutischen T-Zell-Transfer Nebenwirkungen hervorzurufen.

In der Kontaktzone, der sogenannten immunologischen Synapse, zwischen APC und T-Zelle übermittelt die APC 3 Arten von Signalen an die naive T-Zelle (Abb. 1) [2,3]. Einerseits wird über den TCR-Kontakt eine Aktivierung ausgelöst, die durch die kostimulatorische Bindung der B7-Moleküle aufseiten der APC mit dem CD28-Molekül aufseiten der T-Zelle unterstützt wird und zur Aktivierung der T-Zelle führt. Die Ausschüttung von Zytokinen aus der APC beeinflusst die Differenzierung der TZelle. Nach Aktivierung einer T-Zelle wird nach einiger Zeit eine Bremse aktiviert, um überschießende Immunreaktionen zu verhindern: Zytotoxisches T-Lymphozyten-Antigen-4 (CTLA-4) bindet mit höherer Affinität an B7 und verdrängt so das CD28. Dadurch wird der kostimulatorische Effekt beendet und der Aktivierungsgrad der T-Zelle heruntergeregelt.

Gegen körpereigenes Gewebe besteht eine Immuntoleranz, die zentral über positive und negative Selektion durch den Thymus und peripher über regulatorische T-Zellen (Tregs) sowie über negatives Signaling durch CTLA-4 und den Programmed Death1(PD-1)-Rezeptor und seinen Liganden PD-L1 geregelt wird.

\section{Tumorantigene}

Auch Tumorzellen haben Antigene, die durch Überexpression, De-novo-Expression und Genalterationen entstehen und vom Immunsystem grundsätzlich erkannt werden können [4]. Grundsätzlich kann eine immunologische Antitumor-Reaktion stattfinden, die jedoch durch eine Vielfalt von Einflüssen eingeschränkt und modifiziert wird. Die individuelle antitumorale T-Zell-Antwort ist unter anderem bestimmt durch mutierte Neoantigene, den HLA-Polymorphismus, das T-Zell-Repertoire, periphere und zentrale Immuntoleranz, das Alter und das Expressionsprofil des Tumors. Hinzu kommen Immunbarrieren, die neben Tumoren auch von nichtmalignen Geweben intensiv genutzt werden. Diese Barrieren sind in ihrer Vielfalt, Spezifität und Beeinflussbarkeit bislang nur in ersten Ansätzen erfasst worden.

Die Charakterisierung der Tumorantigene hat die Möglichkeit der Gewinnung und Vermehrung tumorspezifischer T-Lymphozyten für eine adoptive Immuntherapie bis hin zu einer personalisierten Immuntherapie mit gentechnisch modifizierten Zellen erweitert [5]. Die Wirksamkeit dieser Immuntherapie scheint unter anderem mit dem vorhandenen individuellen T-Zell-Repertoire zu korrelieren. So sprachen Melanompatienten auf die Impfung mit Peptiden besser an, wenn ihre T-Zellen eine breitere Reaktivität gegen die Tumorantigene zeigten [6].

\section{Immunoediting}

Das Konzept der Immunosurveillance (Immunüberwachung) von Tumoren geht davon aus, dass Tumorzellen mit ihren Tumorantigenen vom Immunsystem erkannt werden und sich zunächst ein Gleichgewicht zwischen Tumorzellwachstum und Zerstörung der

\section{KARGER}

Fax +49761 4520714

Information@Karger.com

www.karger.com
(C) 2013 S. Karger GmbH, Freiburg

0378-584X/13/3616-0007\$38.00/0 


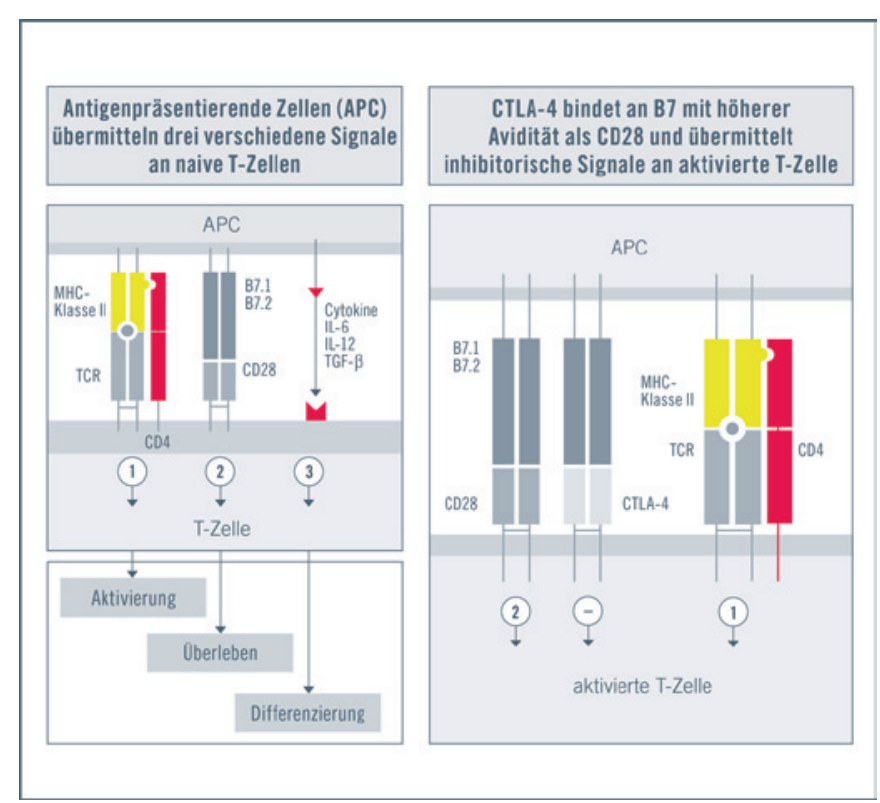

Abb. 1. T-Zell-Aktivierung und -Inhibition (modifiziert nach [2]).

Tumorzellen durch aktivierte Immunzellen einstellt [7]. Unter diesem Selektionsdruck entstehen nicht mehr vom Immunsystem erkennbare Tumorzellen, beispielsweise durch Antigen- und HLA-Verlust. Es spricht aber auch vieles dafür, dass spontan gebildete Tumoren eine Toleranz gegenüber den T-Zellen induzieren, ohne dass die Tumoren ihre intrinsische Immunogenität auf Dauer verlieren $[8,9]$. Nach Transplantation in T-Zell-kompetente Empfänger werden diese Tumorzellen abgestoßen.

\section{Ausblick}

Zurzeit werden verschiedene Strategien zur Verbesserung der Immuntherapie in der Onkologie untersucht. Bei der aktiven zellulären Immuntherapie geht es um die Überwindung immunologischer Barrieren durch Unterdrückung von regulatorischen $\mathrm{T}$ Zellen, Ausschalten des negativen Signalings, Anti-Angiogenese, Inhibition des transformierenden Wachstumsfaktors $\beta$ (TGF- $\beta$ ) sowie durch Chemotherapie und Strahlentherapie, die ebenfalls zum immunogenen Zelltod führen und in Kombination mit einem Immuntherapeutikum die Immunantwort verstärken können. Der adoptive Transfer soll durch die Verwendung von TZellen mit hoher Spezifität und Stammzellcharakter verbessert werden [10].

\section{Immunonkologie beim Melanom: Grenzen und Chancen der Therapie}

Immunaktivierende Anti-CTLA-4-Antikörper setzen bei einem wichtigen Kennzeichen von Tumoren an: Sie verhindern, dass sich Krebszellen der Zerstörung durch das Immunsystem entziehen, und gehören zu einem neuen Tumorkennzeichen (hallmark of cancer) [11]. Der Anti-CTLA-4-Antikörper Ipilimumab ist seit 2011 für die Behandlung von Patienten mit fortgeschrittenem (nicht resezierbarem und metastasiertem) Melanom zuge-

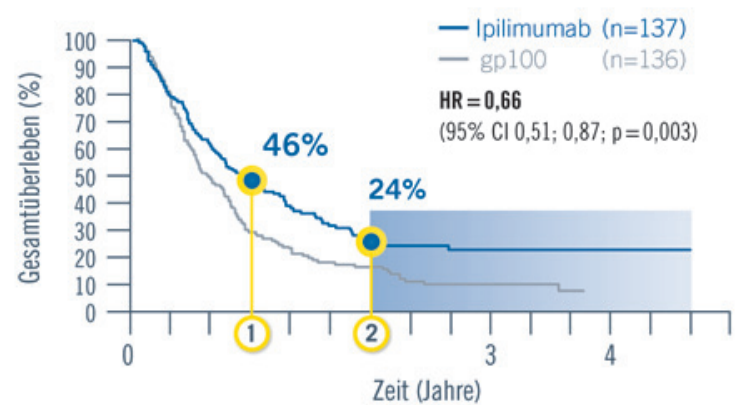

Abb. 2. Zulassungsstudie MDX010-20: Lang andauernder Überlebensvorteil mit Ipilimumab (modifiziert nach [13]).

lassen. Die Induktionstherapie mit Ipilimumab umfasst 4 Infusionen à $3 \mathrm{mg} / \mathrm{kg}$, die im Abstand von jeweils 3 Wochen appliziert werden. Die Blockade von CTLA-4 durch Ipilimumab hebt die Inhibition des kostimulatorischen Signals auf und führt zur Proliferation der aktivierten zytotoxischen T-Zellen und nachfolgend $\mathrm{zu}$ einer gegen das Melanom gerichteten induzierten Immunantwort [12].

\section{CTLA-4-Blockade}

Dass der immunmodulatorische Ansatz mit Ipilimumab bei vorbehandelten Patienten mit fortgeschrittenem Melanom wirkt, belegte die Zulassungsstudie MDX010-20 [13]. In dieser Phase-IIIStudie verlängerte der Anti-CTLA-4-Antikörper signifikant das Gesamtüberleben im Vergleich zur Vakzinierung mit gp100 (glycoprotein 100) von 6,4 auf 10,1 Monate $(\mathrm{p}=0,003)$ und verbesserte das 1- und 2-Jahres-Überleben von $25 \%$ auf $46 \%$ bzw. von $14 \%$ auf $24 \%$ (Abb. 2).

Mittlerweile liegt eine Metaanalyse von 15 Studien vor, in denen das Gesamtüberleben und der Benefit der Ipilimumab-Induktionstherapie bei vorbehandelten Patienten mit fortgeschrittenem Melanom mit anderen Mono- und Kombinationstherapien verglichen wurden [14]. Das mittlere Gesamtüberleben unter Ipilimumab betrug 18,8 Monate und war jeweils länger im Vergleich zu einer Mono-Chemotherapie (Dacarbazin oder Paclitaxel 12,3 Monate), verschiedenen Chemotherapie-Kombinationen (z.B. Dacarbazin plus Cisplatin, Carmustin und Tamoxifen oder Paclitaxel plus Carboplatin 12,2 Monate), Biochemotherapie mit und ohne Chemotherapie (Interleukin(IL)-2 plus Interferon(IFN)alpha plus verschiedene Chemotherapeutika 11,9 Monate), einer Mono-Immuntherapie (gp100 oder IL-2 11,1 Monate) und einer kombinierten Immuntherapie (IL-2 plus IFN-alpha 14,1 Monate) (Abb. 3).

Wie bei anderen Immuntherapeutika auch, beispielsweise Hochdosis-IL-2, wirkt Ipilimumab indirekt über die Aktivie- 


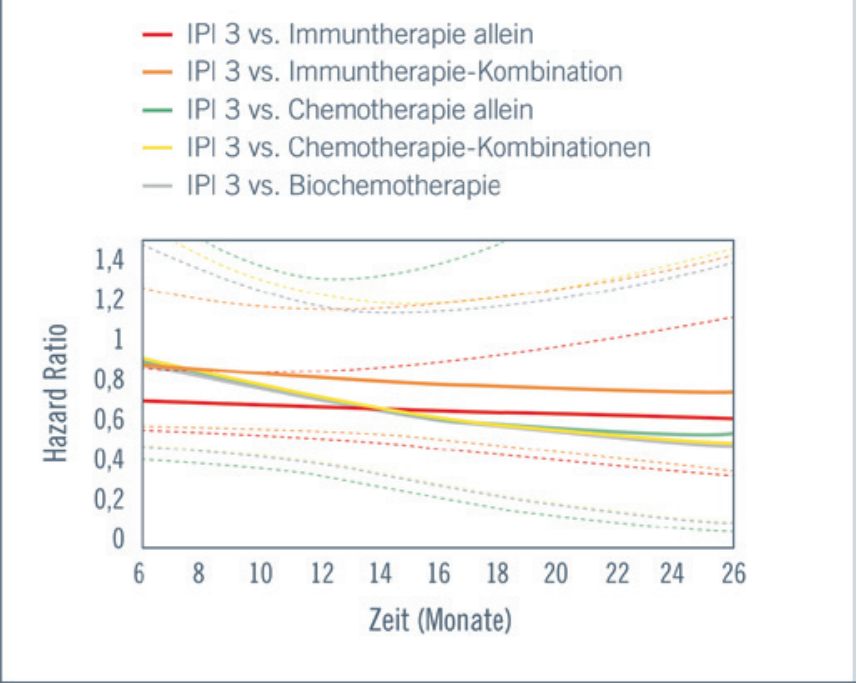

Abb. 3. Gesamtüberleben Ipilimumab (3 mg/kg, IPI 3) im Vergleich zu verschiedenen Chemo- und Immuntherapien (modifiziert nach [14]).

rung des Immunsystems. Das Ansprechmuster unter Ipilimumab unterscheidet sich deshalb im zeitlichen Verlauf, der Dauer und im klinischen Bild von den konventionellen Ansprechmustern von Chemotherapeutika und Kinaseinhibitoren bei soliden Tumoren. Die sogenannten immunvermittelten Ansprechkriterien (immune-related response criteria, irRCs) sind mittlerweile in klinische Studien eingeflossen [15]. Auch das Nebenwirkungsprofil von Ipilimumab belegt dessen immunmodulatorische Wirkweise. Ipilimumab kann immunvermittelte Nebenwirkungen (immune-related adverse events, irAEs) hervorrufen, die autoimmun vermittelten Entzündungssymptomen ähneln [16]. Bei zeitnaher Beachtung der für diese Immuntherapie entwickelten Behandlungsalgorithmen, in der Regel mit Kortikosteroiden und gegebenenfalls weiteren Immunsuppressiva, sind diese Nebenwirkungen üblicherweise gut beherrschbar. Die Algorithmen zum Management der irAEs unter Ipilimumab stehen mittlerweile als kostenlose App zur Verfügung.

\section{PD-1/PD-L1-Blockade}

Eine weitere immuntherapeutische Zielstruktur in der immunologischen Synapse ist die Blockade des PD-1-Rezeptors. Der PD1-Rezeptor bremst ebenso wie CTLA-4 die T-Zellaktivierung nach Antigenstimulation, allerdings im Unterschied zu CTLA-4 nicht durch die sekundäre Hemmung eines kostimulatorischen Effekts, sondern durch einen primären inhibitorischen Mechanismus. PD-1 wirkt vor allem im peripheren Gewebe durch die Bindung mit seinem immunsuppressiven Liganden PD-L1, der von den Tumorzellen gebildet wird. Kann PD-L1 nicht an PD-1 binden, unterbleibt die T-Zell-Inaktivierung. Es liegen bereits erste vielversprechende Studienergebnisse mit einem Anti-PD-1- und einem Anti-PD-L1-Antikörper bei Patienten mit verschiedenen fortgeschrittenen soliden Tumoren vor [17].

\section{Klassische Chemotherapie}

Auch Chemotherapeutika können antitumorale Immunantworten auslösen. In mehreren humanen Melanom-Zelllinien konnte z.B. durch Dacarbazin oder Cisplatin in vitro die Ausschüttung von T-Zell-affinen Chemokinen induziert werden [18]. Identifiziert wurden die zu den Chemokinen gehörenden CXCR3-Liganden und CCL5, die zu einer T-Zell-Infiltration in kutane Tumoren und nachfolgend zu einem Stopp des Tumorwachstums führten. In einer anderen Studie konnte in kutanen Tumoren von einigen Melanompatienten nach der Behandlung mit Dacarbazin eine Hochregulierung von immunassoziierten Genen nachgewiesen werden: Durch die Veränderung des Tumorstromas wanderten mehr T-Zellen, vor allem CD8+-Zellen, in den Tumor ein, was mit einem klinischen Ansprechen korrelierte [19].

\section{Biomarker}

Nicht alle Melanompatienten profitieren von einer Immuntherapie. Bisher gibt es jedoch keinen Nachweis eines prädiktiven Biomarkers für eine Immuntherapie bei Melanompatienten aus einer großen randomisierten Studie. Die existierenden Anhaltspunkte zu Biomarkern bei Melanompatienten wurden im Wesentlichen aus retrospektiven Untersuchungen oder aus der Analyse von kleinen Subgruppen gewonnen.

Ein prädiktiver Marker für das Überleben von Melanompatienten unter einer Immuntherapie könnte der in Europa relativ häufige CCR5-delta-32-Polymorphismus $(\mathrm{CCR} \Delta 32)$ sein [20]. Der CCR5-Rezeptor ist für die Migration von T-Zellen verantwortlich. Die CCRA32-Mutation resultiert in einem nicht funktionierenden Protein und ist beispielsweise auch für eine Resistenz gegen das humane Immundefizienz-Virus (HIV) verantwortlich. Die Analyse von 782 Serumproben von Melanompatienten ergab bei $11,5 \%$ einen heterozygoten und bei $1,5 \%$ einen homozygoten CCRA32-Polymorphismus. Auf das krankheitsspezifische Überleben in der Gesamtkohorte hatte der Polymorphismus keinen Einfluss, auch nicht auf das Therapieansprechen im Stadium III. In der Gruppe der 139 Melanompatienten im Stadium IV, die aber eine Immuntherapie erhielten, war das Überleben bei Patienten mit CCR $\Delta 32$ schlechter als ohne CCR $\Delta 32$.

Ist vor der Therapie bereits eine Immunaktivierung im Tumormikromilieu nachweisbar, scheinen die Patienten besser auf eine Immuntherapie anzusprechen [21, 22]. Die absolute Lymphozytenzahl im peripheren Blut hat wahrscheinlich auch einen positiven Effekt auf das Ansprechen einer Immuntherapie mit Ipilimumab [23]. Auch ein höherer Anstieg der absoluten Lymphozytenzahl nach 2 Ipilimumab-Infusionen korrelierte mit einer besseren immunologischen antitumoralen Wirksamkeit [24].

\section{Ausblick}

Neben der Blockade von Checkpoint-Molekülen wie CTLA-4 und PD-1 mit seinem Liganden PD-L1 werden in der Immuntherapie des Melanoms weitere immuntherapeutische Zielstrukturen untersucht. Dazu gehört beispielsweise die Aktivierung von CD40 und CD137, die in der Antitumor-Immunantwort eine wichtige Rolle spielen. Weitere experimentelle immuntherapeutische Ansätze sind die Vakzinierung mit IL-2, die Therapie mit dem Immunzytokin L19-IL2 in Kombination mit Dacarbazin und die adoptive Immuntherapie. 


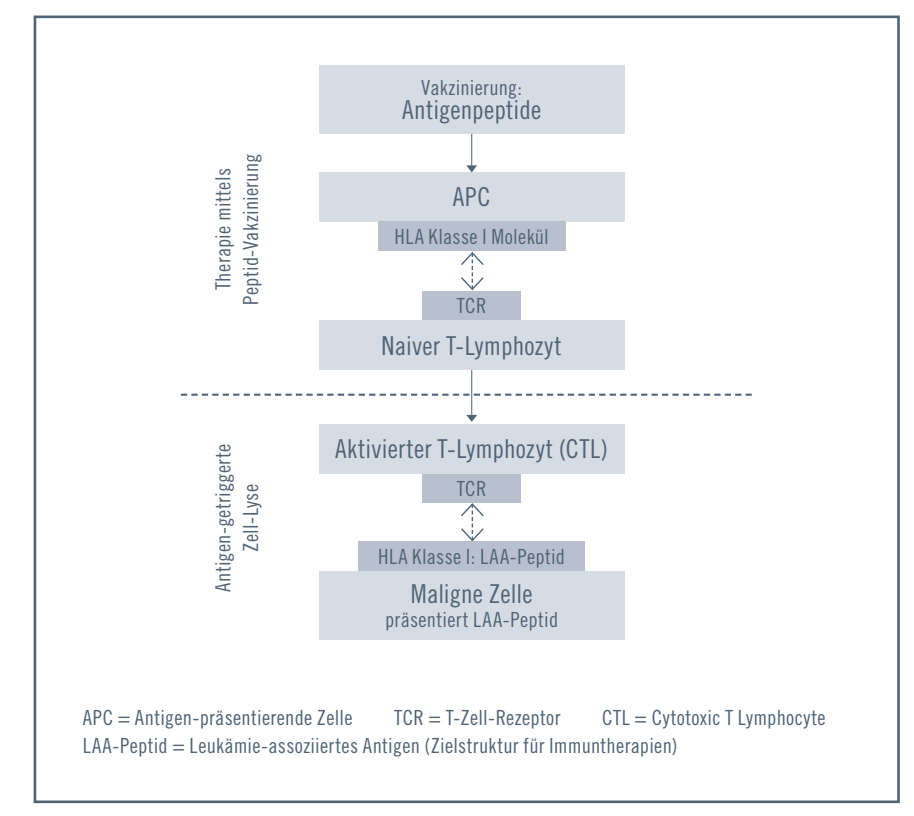

Abb. 4. Konzept der Peptid-Vakzinierung (modifiziert nach [30]).

\section{Immunonkologie bei anderen soliden Tumoren: Fiktion oder begründeter Anlass zur Hoffnung?}

Die Immuntherapie hat mittlerweile bei der Therapie von verschiedenen soliden Tumoren Fortschritte erzielt. Neben der CTLA-4-Blockade beim fortgeschrittenen Melanom steht auch für das Prostatakarzinom eine Immuntherapie zur Verfügung. Seit 2010 ist - allerdings nur in den USA - für asymptomatische oder minimal symptomatische Patienten mit hormonrefraktärem Prostatakarzinom das Immuntherapeutikum Sipuleucel-T zugelassen [25]. Die therapeutische Vakzinierung mit Sipuleucel-T muss auf den einzelnen Patienten angepasst werden.

Zurzeit laufen Phase-III-Studien, die die Wirksamkeit einer therapeutischen Vakzinierung beim Melanom und beim nichtkleinzelligen Lungenkarzinom (NSCLC) untersuchen. Das MAGE(melanoma-associated antigen)-A3-Antigen wird in Tumorzellen des Melanoms, vor allem des metastasierten Melanoms, und des NSCLC exprimiert [26]. Eine Phase-II-Studie mit MAGE-3 zeigte vielversprechende Ergebnisse, eine Phase-IIIZulassungsstudie wird voraussichtlich Mitte 2013 abgeschlossen sein. Zurzeit wird die MAGE-A3-Vakzinierung mit dem Adjuvans AS-15 (antigen-specific cancer immunotherapy, ASCI) beim Melanom und NSCLC untersucht. In der Studie DERMA sollen insgesamt 1300 MAGE-A3-positive Melanompatienten im Stadium III adjuvant im Verhältnis 2:1 mehrere ASCI-Impfungen oder Placebo erhalten [27].

Ein neuer immuntherapeutischer Ansatz in der Hämatologie ist das Klonen von chimären antigenspezifischen Rezeptoren in die T-Zelle hinein, um eine hohe Spezifität zu erreichen. T-Zellen werden gentechnisch mit Rezeptoren ausgestattet, über die sie das Protein CD19 auf der Oberfläche von B-Zellen erkennen können (chimärische Antigen-Rezeptor-T-Zellen, die gegen CD19 gerichtet sind, CART19). CD19 ist bei B-Zell-Lymphomen eine attraktive Zielstruktur. Mit CART19 konnte bei einem Patienten mit chronischer lymphatischer Leukämie (CLL) die Krankheit in Remission gebracht werden [28].

\section{Peptid-Vakzinierung}

In der Hämatologie werden in den immuntherapeutischen Ansätzen möglichst spezifisch Antigene der malignen Zelle anvisiert und keine Strukturen des peripheren Blutes wie Granulozyten, Monozyten oder Lymphozyten sowie keine CD34+-Stammzellen der normalen Hämatopoese. Mit dem molekularbiologischen Verfahren der serologischen Identifizierung von Antigenen durch rekombinantes Expressionsklonieren (SEREX) ist eine serologische Identifikation von Antigenen möglich.

Mittels SEREX wurde RHAMM (receptor for hyaluronic acid-mediated motility) definiert, der bei akuten und chronischen Leukämien und beim multiplen Myelom (MM) exprimiert ist [29]. RHAMM sitzt am Spindelapparat der Zelle und ist maßgeblich an der Polarität für die Zellteilung beteiligt. In soliden Tumoren geht die RHAMM-Expression mit Tumorprogress und Metastasierung einher, beim Melanom kommt RHAMM nur in den Metastasen und nicht im Primärtumor vor. Das von RHAMM abgeleitete stark immunogene CD8+ T-Zell-EpitopPeptid R3 (ILSLELMKL) wird zurzeit zur Peptid-Vakzinierung bei Leukämien experimentell eingesetzt.

Bei der Peptid-Vakzinierung werden subkutan Antigenpeptide mit einer Antigenpeptid-Spritze eingebracht, sodass das Peptid auf ein HLA-Klasse-I-Molekül trifft (Abb. 4) [30]. Dadurch werden naive T-Zellen zu aktivierten CTLs «geprimt», die das Antigen auf Tumorzellen erkennen und zur Antigen-getriggerten Zelllyse der Tumorzellen führen.

Die ILSLELMKL-Vakzinierung von 10 Patienten mit akuter myeloischer Leukämie (AML), myelodysplastischem Syndrom (MDS) oder MM induzierte bei 7 Patienten einen immunologischen und klinischen Effekt [31]. Die Patienten erhielten 4 Vakzinierungen mit dem RHAMM-Peptid mit jeweils 2 Wochen Abstand. Im Gegensatz zu anderen Peptid-Vakzinierungsstudien zeigte sich eine gute Konkordanz von immunologischem und klinischem Ansprechen. Die Peptid-Vakzine wird subkutan injiziert. Als Nebenwirkungen traten lediglich Rötungen an der Einstichstelle auf.

\section{Ausblick}

Impfungen und Immuntherapien können durch die Auswahl von besseren Zielstrukturen, die Verwendung von effektiveren Adjuvantien, die Blockade von hemmenden Signalen wie CTLA-4, PD-1 und PD-L1 und regulatorischen Zellen, aber auch durch die Kombination immuntherapeutischer Strategien verbessert werden. Dabei ist zu beachten, dass Kombinationen außer den gewünschten Wirkungen auch unerwünschte Nebenwirkungen zeitigen.

\section{Disclosure Statement}

Die Autoren waren Referenten beim «3. Interdisziplinären Expertenforum Hautkrebs», das von Bristol-Myers Squibb veranstaltet und unterstützt wurde. 


\section{Literatur}

1 Murphy K, Travers P, Walport M, et al.: Janeway's Immunobiology, ed 7. New York, Garland Science, 2008

2 Murphy K, Travers P, Walport M, et al.: Janeway Immunologie, ed 7. Heidelberg, Spektrum Akademischer Verlag, 2010

3 Graykoui A, Bromley SK, Sumen C, et al.: The immunological synapse: a molecular machine controlling T cell activation. Science 1999;285:221-227.

4 van der Bruggen $\mathrm{P}$, Stroobant V, Vigneron $\mathrm{N}$, et al. T-cell defined tumor antigens. http://cancerimmunity. org/peptide/.

$\checkmark 5$ Rosenberg SA: Raising the bar: the curative potential of human cancer immunotherapy. Sci Trans Med 2012;4:127-135.

6 Walter A, Weinschenk T, Stenzl A, et al.: Multipeptide immune response to cancer vaccine IMA901 after single-dose cyclophosphamide associates with longer patient survival. Nat Med 2012; DOI: $10.1038 / \mathrm{nm} .2883$

7 Dunn GP, Bruce AT, Ikeda H, et al.: Cancer immunoediting: from immunosurveillance to tumor escape. Nat Immunol 2002;3:991-998.

8 Willimsky G, Blankenstein T: Sporadic immunogenic tumors avoid destruction by inducing $\mathrm{T}$ cell tolerance. Nature 2005;437:141-146.

9 Landsberg J, Kohlmeyer J, Renn M, et al.: Melanomas resist T-cell therapy through inflammationinduced reversible dedifferentiation. Nature 2012; 490:412-416.

10 Gattinoni L, Klebanoff CA, Restifo NP: Paths to stemness: building the ultimate antitumour T cell. Nat Rev Cancer 2012;12:671-684.

11 Hanahan D, Weinberg RA: Hallmarks of cancer: the next generation. Cell 2011;144:646-674.

12 Chambers CA, Kuhns MS, Jackson GE, et al. CTLA-4-mediated inhibition in regulation of $\mathrm{T}$ cell responses: mechanisms and manipulation in tumor immunotherapy. Ann Rev Immunol 2001;19:565594.
13 Hodi FS, Steven J, O'Day MD, et al.: Improved survival with ipilimumab in patients with metastatic melanoma. N Engl J Med 2010;363:711-723.

14 Dequen P, Lorigan P, Jansen JP, et al.: Systematic review and network meta-analysis of overall survival comparing $3 \mathrm{mg} / \mathrm{kg}$ ipilimumab with alternative therapies in the management of pretreated patients with unresectable stage III or IV melanoma. Oncologist 2012;17:1376-1385.

15 Hoos A, Eggermont AM, Janetzli S, et al.: Improved endpoints for cancer immunotherapy trials. J Natl Cancer Inst 2010;102:1388-1397.

16 Fachinformation Yervoy ${ }^{\circledR}$

17 Allison J: Mobilizing the immune system to treat cancer: immune checkpoint strategy. American Society of Clinical Oncology (ASCO) Meeting 2012; Clinical Science Symposium 'Immune Checkpoint Strategies'.

18 Hong M, Puaux AL, Huang C, et al.: Chemotherapy induces intratumoral expression of chemokines in cutaneous melanoma, favoring $\mathrm{T}$ cell infiltration and tumor control. Cancer Res 2011;71:6997-7009.

19 Nardin A, Wong, Tow C, et al.: Dacarbazine promotes stromal remodeling and lymphocyte infiltration in cutaneous melanoma lesions. J Invest Dermatol 2011;131:1896-1905.

20 Ugurel S, Schrama D, Keller G, et al.: Impact of the CCR5 gene polymorphism on the survival of metastatic melanoma patients receiving immunotherapy. Cancer Immunol Immunother 2008;57: 685-691.

21 Hamid O, Schmidt H, Nissan A, et al.: A prospective phase II trial exploring the association between tumor microenvironment biomarkers and clinical activity of ipilimumab in advanced melanoma. J Transl Med 2011;9:204-220.

22 Ji RR, Chasalow SD, Wang L, et al.: An immuneactive tumor microenvironment favors clinical response to ipilimumab. Cancer Immunol Immunother 2012;61:1019-1031.
$23 \mathrm{Ku}$ GY, Page DB, Schroeder SE, et al.: Single-institution experience with ipilimumab in advanced melanoma patients in the compassionate use setting: lymphocyte count after 2 doses correlates with survival. Cancer 2010;116:1767-1775.

24 Berman DM, Wolchok J, Weber J, et al.: Association of peripheral blood absolute lymphocyte count (ALC) and clinical activity in patients (pts) with advanced melanoma treated with ipilimumab. J Clin Oncol 2009;27(15s):abstr 3020.

25 Food and Drug Administration (FDA), www.fda. gov/BiologicsBloodVaccines/CellularGeneTherapyProducts/ApprovedProducts/ucm210012.htm.

26 Roeder C, Schuler-Thiurner B, Berchtold S, et al.: MAGE-A3 is a frequent tumor antigen of metastasized melanoma. Arch Dermatol Res 2005;296:314 319.

27 Kirkwood JM, Dreno B, Hauschild A, et al. DERMA phase III trial of MAGE-A3 antigenspecific cancer immunotherapeutic (ASCI) as adjuvant therapy in patients with MAGE-A3-positive resected stage III melanoma. American Society of Clinical Oncology (ASCO) Meeting 2011;abstr TPS232.

28 Porter DL, Levine BL, Kalos M, et al.: Chimeric antigen receptor-modified $\mathrm{T}$ cells in chronic lymphoid leukemia. N Engl J Med 2011;365:725-733.

29 Greiner J, Ringhoffer M, Taniguchi M, et al.: Receptor for hyaluronan acid-mediated motility (RHAMM) is a new immunogenic leukemia-associated antigen in acute and chronic myeloid leukemia. Exp Hematol 2002;30:1029-1035.

30 Schmitt M, Casalegno-Garduno R, Xu X, Schmitt A: Peptide vaccines for patients with acute myeloid leukemia. Expert Rev Vaccines 2009;8:1415-1425.

31 Schmitt M, Schmitt A, Rojewski MT, et al.: RHAMM-R3 peptide vaccination in patients with acute myeloid leukemia, myelodysplastic syndrome, and multiple myeloma elicits immunologic and clinical responses. Blood 2008;111:1357-1365. 